\section{A computer program to evaluate paired-comparison transitivity}

\section{EDWARD BURNS \\ School af General Studies and Professional Education State University of New York, Binghamton, New York 13901}

The method of paired comparisons is a useful assessment technique when a simple task is required or when the underlying transitivity of elements being compared is suspect. The transitivity of elements can be assessed by evaluating all possible triples or, where $n$ signifies the number of elements being compared, $n(n-1)(n-2) / 6$ triples. The number of transitive triples (e.g., $A>B, B>C, A>C$ ) can be determined from the row marginals of the pairedcomparison scoring matrix (see Harary, Norman, \& Cartwright, 1965), but determining exactly which triples are transitive or cyclic $(\mathrm{A}>\mathrm{B}, \mathrm{B}>\mathrm{C}, \mathrm{C}>\mathrm{A})$ can be a formidable task. When $\mathrm{n}$ is 5 , the number of triples is a manageable 10 , but when $n$ is 10 , the number of triples is 120 , and when $\mathrm{n}$ is 20 , the number of triples is 1,140 .

The present program, written in FORTRAN IV, evaluates all possible triples, prints all triples that are cyclic, and provides several statistics relating to transitivity.

Input. The first card indicates the size (i.e., order) of the paired-comparison scoring matrix (n can range from 3 to 25), followed by cards representing the paired-comparison scoring matrix; each card signifies one row of the matrix. In a paired-comparison scoring matrix, an entry of 1 in the ith row and jth column indicates that the ith element was preferred to the jth element; an entry of 0 indicates the opposite.

Output. The output consists of the printing of input data, followed by a listing of all cyclic triples and indexes relating to transitivity. The following is an example of a cyclic triple:

$$
\text { 4 P 7, } 7 \text { P 9, BUT } 9 \text { P 4, }
$$

where P indicates that the ith element was "preferred" to the jth element.

The statistics produced consist of the total number of triples, the possible number of cyclic triples, the actual number of cyclic triples, the number of cyclic triples expected by chance (which is .25 the number of total triples possible), and an index of transitivity (see Kendall \& Smith, 1940) that is calculated by 1 - (actual cyclic triples/possible cyclic triples). An error message is printed and the program is terminated if the matrix size is beyond the specified limit (25), if a matrix entry is other than 0 or 1 , or if offdiagonal corresponding row and column entries are equal such that $M(I, J)=M(J, I)$. If no errors occur, as many matrices as specified will be evaluated.

Availability. A listing of the FORTRAN IV program and sample input and output data are available at no cost from Edward Burns, School of General Studies and Professional Education, State University of New York, Binghamton, New York 13901.

\section{REFERENCES}

Harary, F., Norman, R. Z., \& Cartwright, D. Structural models: An introduction to the theory of directed graphs. New York: Wiley, 1965.

Kendali, M. G., \& SMith, B. B. On the method of paired comparisons. Biometrika, 1940, 31, 324-345.

(Accepted for publication December 1, 1980.) 